DESY98-198

RUB-TPII-20/98

\title{
Hard exclusive pseudoscalar meson electroproduction and spin structure of a nucleon
}

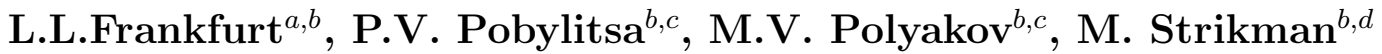 \\ ${ }^{a}$ Physics Department, Tel Aviv University, Tel Aviv, Israel \\ ${ }^{b}$ Petersburg Nuclear Physics Institute, Gatchina, Russia \\ ${ }^{c}$ Institut für Theoretische Physik II, Ruhr-Universität Bochum, \\ D-44780 Bochum, Germany \\ ${ }^{d}$ Department of Physics, Pennsylvania State University, University Park, PA 16802, \\ USA, \\ and Deutsches Elektronen Synchrotron DESY, Germany ${ }^{1}$
}

\begin{abstract}
The amplitude for hard exclusive pseudoscalar meson electroproduction off nucleon (nuclear) targets is computed in QCD within the leading $\alpha_{s} \ln Q^{2} / \lambda_{Q C D}^{2}$ approximation. We show that the distribution of recoil nucleons depends strongly on the angle between the momentum of the recoil nucleon and the polarization vector of the target (or outgoing nucleon). This dependence is especially sensitive to the spin flip skewed parton distribution (SPD) $\widetilde{E}$. We argue also that the scaling for this spin asymmetry sets in at lower $Q^{2}$ than that for the absolute cross section. Basing on the chiral quark-soliton model of the nucleon we estimate quantitatively the spin asymmetry. In the case of $\pi^{+}$production this asymmetry is dominated at small $t$ by the contribution of the pion pole in the isovector SPD $\widetilde{E}$ as required by PCAC. In the case of $K^{0}$ production off a proton we find a large enhancement of the cross section as compared to the case of $\pi^{0}$ production. For the forward production of neutral pseudoscalar mesons off a deuteron target we find the cross section should be zero for the zero deuteron helicity (along the $\gamma^{*} D$ direction). We consider also cross sections of quasielastic processes off nuclei including the feasibility to implant $K^{+}, \rho$-mesons into nuclear volume.
\end{abstract}

\section{Introduction}

Hard exclusive electroproduction of mesons is a new kind of hard process calculable in QCD. In [1] the QCD factorization theorem was proven for the process

$$
\gamma_{L}^{*}(q)+p \rightarrow M(q-\Delta)+B^{\prime}(p+\Delta)
$$

at large $Q^{2}$, with $t$ and $x=Q^{2} / 2 p \cdot q$ fixed. Here $M$ is any meson with mass $m$ satisfying the condition: $Q^{2} \gg m^{2}$. The factorization theorem asserts that the amplitude has the form

$$
\begin{aligned}
& \sum_{i, j} \int_{0}^{1} d z \int d x_{1} f_{i / p}\left(x_{1}, x_{1}-x ; t, \mu\right) H_{i j}\left(Q^{2} x_{1} / x, Q^{2}, z, \mu\right) \phi_{j}(z, \mu) \\
& \text { +power-suppressed corrections, }
\end{aligned}
$$


where $f_{i / p}$ is a skewed parton distribution (SPD) [2, 3, 田, 6, 1, 6, 7, 8], $x_{1}$ is the fraction of the target momentum carried by the interacting parton, $\phi_{j}$ is the light-front wave function of the meson, and $H_{i j}$ is a hard-scattering coefficient, computable in powers of $\alpha_{s}(Q)$. Qualitatively one can say that these reactions allow one to perform a " microsurgery" of a nucleon by removing in a controlled way a quark of one flavor and spin and implanting instead another quark (in general with a different flavor and spin). Use of the QCD factorization theorem makes it possible to predict the absolute value of the cross section at sufficiently large $Q^{2} \geq 10 \mathrm{GeV}^{2}$ and sufficiently small $x$ where skewed parton distributions in a nucleon are calculable in QCD [9, 10]. For example, the exclusive neutral pion production is calculable in terms of the valence quark distribution of nucleon spin 11, 11, 12]. (An educated guess is that the region of applicability of the leading twist contribution to hard exclusive processes should be close to that for the electromagnetic form factor of the pion. Thus we expect that $Q^{2} \geq 10-15 \mathrm{GeV}^{2}$ should be a good estimate, cf. discussion of the pion electromagnetic form factors in [13, 14]) and that approach to the scaling limit for pole contribution would happen from above.

In this paper we derive general formulae for the amplitude of the hard exclusive production of pseudoscalar mesons off nucleons and nuclei and focus on the calculation of the dependence of the cross section on the polarization of the target nucleon which should be much less sensitive to the inputs used in the calculation. Really, an experimental investigation of the spin asymmetries discussed in the paper as well as ratios of the yields of $\eta^{\prime}, \eta, \pi^{0}$ investigated in [15], and $K / \pi$ considered in this paper has an important advantage. In fact, it was demonstrated in [16] that up to rather large $Q^{2}$ the absolute cross sections of hard exclusive processes are suppressed significantly due to the higher twist effects originating from the comparable transverse size of the longitudinal photon and the meson wave functions. However the overall transverse size was found to be sufficiently small $\leq 0.4 \mathrm{fm}$ at $Q^{2} \geq 5 \mathrm{GeV}^{2}$. Due to the color transparency phenomenon this leads to a strong suppression of the final state interaction of the $q \bar{q}$ pair which in the end will form the meson and the residual baryon system. For $W \leq 20 \mathrm{GeV}$ this cross section is of the order of few mb. Besides, the expansion of the $q \bar{q}$ system to a normal hadron size in the nucleon rest frame takes a distance $l_{c o h} \sim 2 p_{M} / \Delta m_{M}^{2} \sim \frac{1}{x M_{N}} Q^{2} / \Delta m_{M}^{2}$ where $\Delta m_{M}^{2}$ is the characteristic light-cone energy denominator for a meson $M$ which is $\leq 1 \mathrm{GeV}^{2}$, cf. [17]. One can easily see that the condition $l_{\text {coh }} \gg r_{N}$ is satisfied for $x \leq 0.2$ already for $Q^{2} \geq 5 \mathrm{GeV}^{2}$. Hence, it seems likely that a precocious factorization into three blocks overlap integral between photon and pseudoscalar meson wave functions, the hard blob, and the skewed distribution - could be valid already at moderately high $Q^{2}$ leading to a precocious scaling of the spin asymmetries and of the ratios of the cross sections as a function of $Q^{2}$. At the same time predictions for the absolute cross sections should be valid only for $Q^{2} \geq 10 \mathrm{GeV}^{2}$.

It is worth emphasizing also that the onset of the hard regime in the case of pion electroproduction (as well as the other channels for which the vacuum exchange is forbidden) can be established via a study of the change of the $t$ dependence with an increase of $Q^{2}$. For the production of electrically neutral pseudoscalar mesons the $t$ dependence should asymptotically approach with an increase of $Q^{2}$ to the value given by the nucleon axial form factor. Besides due to slowing down of the Gribov diffusion in the hard regime one expects a significant decrease of the shrinkage of the forward cone with the incident 
energy at fixed $Q^{2}$. This effect would be easier to detect than in the case of the vector meson production since the slope of the pion trajectory is four times larger than that of the Pomeron trajectory [18].

The paper is organized as following. In section 2 we derive general expressions for the process of pseudoscalar meson production off nucleons. We relate the skewed proton $\rightarrow$ proton transitions to those arising for parton densities for the flavor changing transitions and give predictions for the ratios of production of kaons and pions. In particular we demonstrate that in contrast to the case for soft physics where production of strange particles is suppressed in the hard exclusive processes strange meson production is often enhanced as compared to the pion production. In particular we predict that for small enough $x$ the cross section of the process $\gamma_{L}^{*}+p \rightarrow K^{0}+\Sigma^{+}$should be three times larger than the cross section for the process $\gamma_{L}^{*}+p \rightarrow \pi^{0}+p$. In section 3 we derive general expressions for the dependence of the meson production cross section on the transverse proton polarization (or polarization of the recoil baryon) in terms of the skewed distributions. In section 4 we use the chiral quark soliton model of the nucleon [19] to calculate the relevant skewed distributions and to calculate the polarization in the case of $\pi^{+}$production, and find it to be large due to the contribution of the term controlled by PCAC. In section 5 we consider exclusive pion production off nuclei. We find a number of spin effects for these processes, and explain that color coherence phenomena should be present for these processes. In section 6 we introduce a new class of processes for which the factorization theorem is applicable - exclusive production of leading baryons and antibaryons. We discuss some general features of these processes and suggest a way to use these processes to look for gluonium states and to implant mesons in nuclei.

We present the conclusions in section 7 .

\section{Amplitude for the hard pseudoscalar meson elec- troproduction}

In this section we compute the leading $1 / Q^{2}$ amplitude of the hard pseudoscalar meson electroproduction. This amplitude owing to the factorization theorem for exclusive hard reactions [1] can be written as a convolution of a skewed parton distribution in the nucleon, a distribution amplitude for the produced meson and the hard part computable in pQCD as a series in powers of $\alpha_{s}$. We consider the processes of hard electroproduction of a non-singlet pseudoscalar meson $P=\pi, K$, etc.:

$$
\gamma_{L}^{*}(q)+B_{1}(p) \rightarrow P\left(q^{\prime}\right)+B_{2}(p+\Delta)
$$

where $\Delta=p^{\prime}-p$ is a four momentum transfer to a target baryon $B_{1},-q^{2}=Q^{2} \rightarrow \infty$, $2 p \cdot q \rightarrow \infty$ with $x=Q^{2} / 2 p \cdot q$ fixed.

The corresponding leading order amplitude has the form:

$$
\left\langle B_{2}\left(p^{\prime}\right), P\left(p+q-p^{\prime}\right)\left|J^{\mathrm{e} . \mathrm{m} .} \cdot \varepsilon_{L}\right| B_{1}(p)\right\rangle=-\left(e 4 \pi \alpha_{s}\right) \frac{N_{c}^{2}-1}{N_{c}^{2}} \frac{f_{P}}{4 Q} \int_{-1}^{1} d \tau \int_{0}^{1} d z
$$




$$
\times \sum_{f, f^{\prime}=u, d, s} F_{f f^{\prime}}^{(5)}(\tau, \xi, t) \varphi_{P}^{f^{\prime} f}(z)\left\{\frac{Q_{f^{\prime}}}{z\left(\tau+\frac{\xi}{2}\right)-i 0}+\frac{Q_{f}}{(1-z)\left(\tau-\frac{\xi}{2}\right)+i 0}\right\} .
$$

Here $Q_{f}$ is the charge of a quark of flavor $f=u, d, s$ in units of the proton charge $\left(Q_{u}=2 / 3, Q_{d}=Q_{s}=-1 / 3\right), f_{P}$ is the decay constant of the pseudoscalar meson (e.g. $f_{\pi} \approx 132 \mathrm{MeV}, f_{K} \approx 1.2 f_{\pi}$, etc.), and $\xi$ is a skewedness parameter (see definition below).

In the frame where $\vec{q}=\left(0,0, q_{3}\right)$ the longitudinal polarization vector has the form:

$$
\varepsilon_{L}^{\mu}=\frac{1}{Q}\left(q^{3}, 0,0, q^{0}\right)^{\mu}
$$

$F_{f f^{\prime}}^{(5)}(\tau)$ is a skewed quark distribution defined as:

$$
F_{f f^{\prime}}^{(5)}(\tau, \xi, t)=\int \frac{d \lambda}{2 \pi} e^{i \lambda \tau}\left\langle B_{2}\left(p^{\prime}\right)\left|T\left\{\bar{\psi}_{f^{\prime}}(-\lambda n / 2) \hat{n} \gamma_{5} \psi_{f}(\lambda n / 2)\right\}\right| B_{1}(p)\right\rangle
$$

with the light cone vector $n$ normalized by:

$$
n^{2}=0, \quad n \cdot\left(p+p^{\prime}\right)=2 .
$$

The pseudoscalar meson distribution amplitude $\varphi_{P}^{f^{\prime} f}(z)$ is defined as:

$$
\varphi_{P}^{f^{\prime} f}(z)=\frac{1}{f_{P}} \int \frac{d \lambda}{2 \pi} e^{-i \lambda z\left(q^{\prime} \cdot \widetilde{n}\right)}\left\langle P\left(q^{\prime}\right)\left|T\left\{\bar{\psi}_{f}(\lambda \widetilde{n}) \hat{\tilde{n}} \gamma_{5} \psi_{f^{\prime}}(0)\right\}\right| 0\right\rangle
$$

where for convenience we introduce another light cone vector $\widetilde{n}$ such that $n \cdot \widetilde{n}=1$ and $\widetilde{n}^{2}=0$. Variable $z$ is the longitudinal fraction (along the vector $n$ ) of the meson momentum $q^{\prime}=q+\Delta$ carried by one of the quarks in a meson. Vectors $n$ and $\tilde{n}$ are linear combinations of the photon, initial, and final baryon momenta:

$$
\begin{aligned}
p^{\mu} & =\left(1+\frac{\xi}{2}\right) \widetilde{n}^{\mu}+\left(1-\frac{\xi}{2}\right) \frac{\bar{M}^{2}}{2} n^{\mu}-\frac{1}{2} \Delta_{\perp}^{\mu} \\
p^{\prime \mu} & =\left(1-\frac{\xi}{2}\right) \widetilde{n}^{\mu}+\left(1+\frac{\xi}{2}\right) \frac{\bar{M}^{2}}{2} n^{\mu}+\frac{1}{2} \Delta_{\perp}^{\mu} \\
q^{\mu} & =-\xi \widetilde{n}^{\mu}+\frac{Q^{2}}{2 \xi} n^{\mu} \\
\bar{M}^{2} & =\frac{1}{2}\left(M_{B_{1}}^{2}+M_{B_{2}}^{2}-\frac{\Delta^{2}}{2}\right) .
\end{aligned}
$$

The transverse plane is defined as the plane orthogonal to the plane spanned by the light-cone vectors $n^{\mu}$ and $\widetilde{n}^{\mu}$.

The skewedness parameter $\xi$ (longitudinal component of the momentum transfer) is defined as

$$
\xi=-(n \cdot \Delta) .
$$

In the Bjorken limit when $Q^{2} \rightarrow \infty$ but $x$ is fixed this skewedness parameter $\xi$ is expressed in terms of the Bjorken variable $\xi=\frac{2 x}{2-x}$. 
In the expression (4) a new type of "truly non-diagonal" skewed parton distributions (SPD's) enter; they are given by the transitional matrix element $B_{1} \rightarrow B_{2}$ of a bilocal (generically non-diagonal in the flavor space) quark operator on the light-cone (6). One can use the flavor $S U(3)$ or $S U(2)$ symmetry to relate these distributions to the usual SPD's corresponding to the diagonal transition proton (neutron) $\rightarrow$ proton (neutron), which were introduced to describe the deeply virtual Compton scattering (DVCS) amplitude. One can write for a quark-antiquark operator on a light-cone between two baryons

$$
\left\langle B_{2}\left|\bar{q}_{i} q_{j}\right| B_{1}\right\rangle=F\left[B_{2}^{+}, B_{1}\right]_{j i}+D\left\{B_{2}^{+}, B_{1}\right\}_{j i}+S \delta_{j i} \delta_{B_{2} B_{1}}
$$

Since we are interested in the nondiagonal transitions we can ignore the singlet part. Taking the matrix elements of nonsinglet combinations of $\bar{q}_{i} q_{i}$ operators between the proton states we find:

$$
\begin{gathered}
\langle p|(\bar{u} u-\bar{d} d)=D-F| p\rangle \\
\langle p|(2 \bar{u} u-\bar{d} d-\bar{s} s)| p\rangle=2(D-F)-(D+F)=D-3 F \\
\langle p|(\bar{d} d-\bar{s} s)| p\rangle=-(D+F)
\end{gathered}
$$

Hence in the case of the production of $\pi^{+}$, which is described in terms of the transitional matrix element between proton and neutron we have (the spin indices are suppressed):

$$
\langle n|\bar{d} u| p\rangle=\langle p|\bar{u} u| p\rangle-\langle n|\bar{u} u| n\rangle=\langle p|\bar{u} u| p\rangle-\langle p|\bar{d} d| p\rangle
$$

to relate these new SPD's to the usual ones. The relation (15) and $S U_{f l}(3)$ relations below should be understood as flavor relations between matrix elements (6). They do not imply any relations between, for example, valence and sea quark distributions. For instance, relation (15) states for fixed $\tau$ that:

$$
F_{u d}^{(5)(p \rightarrow n)}(\tau)=F_{u u}^{(5)(p \rightarrow p)}(\tau)-F_{d d}^{(5)(p \rightarrow p)}(\tau) .
$$

Another example is the production of kaons. For instance, $K^{+}$production probes $p \rightarrow \Lambda$ or $p \rightarrow \Sigma^{0}$ transitions in eq. (4). In this case eq.(14) leads to (only flavor quantum numbers are shown) p:

$$
\begin{aligned}
& \langle\Lambda|\bar{s} u| p\rangle=\frac{1}{\sqrt{6}}(3 F-D)=\frac{1}{\sqrt{6}}\langle p|(2 \bar{u} u-\bar{d} d-\bar{s} s)| p\rangle \\
& \left\langle\Sigma^{0}|\bar{s} u| p\right\rangle=\frac{1}{\sqrt{2}}(F+D)=\frac{1}{\sqrt{2}}\langle p|\bar{d} d-\bar{s} s| p\rangle .
\end{aligned}
$$

In the case of $K^{0}$ production from a proton we probe the $p \rightarrow \Sigma^{+}$transition in eq. (幽). The $S U_{f l}(3)$ relation in this case is the following:

$$
\left\langle\Sigma^{+}|\bar{s} d| p\right\rangle=(F+D)=\langle p|\bar{d} d-\bar{s} s| p\rangle .
$$

\footnotetext{
${ }^{2}$ Let us note that although we expect that $S U_{f l}(3)$ relations work rather well for spin nonflip SPD's $\widetilde{H}$, they can be violated rather strongly for "the pole part" of $\widetilde{E}$ (see discussion in section 4). This is due to the rather large mass difference $m_{K}-m_{\pi}$. However the "pole part" is under theoretical control, because it is calculated using PCAC.
} 
We see that the hard electroproduction of pseudoscalar mesons gives the possibility to probe various flavor combinations of polarized quark distributions in the proton and to study in a new way $S U_{f l}(3)$ properties of the strange baryons.

Analogously we can write the flavor decomposition of the pseudoscalar meson distribution amplitudes (8). Here we give some examples for pions and kaons:

$$
\begin{aligned}
\varphi_{\pi^{0}}^{f^{\prime} f}(z) & =\frac{i}{\sqrt{2}}\left[\delta_{u}^{f^{\prime}} \delta_{u}^{f}-\delta_{d}^{f} \delta_{d}^{f^{\prime}}\right] \varphi_{\pi}(z) \\
\varphi_{\pi^{+}}^{f^{\prime} f}(z) & =i \delta_{d}^{f^{\prime}} \delta_{u}^{f} \varphi_{\pi}(z) \\
\varphi_{K^{0}}^{f^{\prime}}(z) & =i \delta_{s}^{f^{\prime}} \delta_{d}^{f} \varphi_{K}(z) \\
\varphi_{K^{+}}^{f^{\prime}}(z) & =i \delta_{s}^{f^{\prime}} \delta_{u}^{f} \varphi_{K}(z)
\end{aligned}
$$

where $\varphi_{\pi}(z)$ and $\varphi_{K}(z)$ are the leading twist pion and kaon distribution amplitudes normalized by the condition: $\int_{0}^{1} d z \varphi_{\pi, K}(z)=1$. In principle, other components of the light cone meson wave function may give a nonzero contribution, but only in subleading order in $1 / Q^{2}$. Thus we took into account in the leading order only the conventional wave function of a pion and a kaon given by the matrix element of the axial current.

The leading twist skewed quark distribution with $f=f^{\prime}=q=u, d, s$ and $B_{1}=$ $B_{2}=$ proton given by eq. (6) can be decomposed into spin nonflip and spin flip parts. Here we adopted the notation of $\mathrm{Ji}$ [6] for the spin decomposition of the matrix element of a bilocal quark operator between protons. The spin nonflip part is denoted as $\widetilde{H}_{q}$ and spin flip as $\widetilde{E}_{q}$. They are defined as:

$$
\begin{aligned}
\int \frac{d \lambda}{2 \pi} e^{i \lambda \tau}\left\langle p^{\prime}\left|\bar{\psi}_{q}(-\lambda n / 2) \hat{n} \gamma_{5} \psi_{q}(\lambda n / 2)\right| p\right\rangle & =\widetilde{H}_{q}(\tau, \xi, t) \bar{U}\left(p^{\prime}\right) \hat{n} \gamma_{5} U(p) \\
& +\frac{1}{2 M_{N}} \widetilde{E}_{q}(\tau, \xi, t) \bar{U}\left(p^{\prime}\right)(n \cdot \Delta) \gamma_{5} U(p)
\end{aligned}
$$

Here $\Delta$ is the four-momentum transfer, $\Delta=p^{\prime}-p, M_{N}$ denotes the nucleon mass, and $U(p), \bar{U}\left(p^{\prime}\right)$ are the standard Dirac spinors. One can chose as independent variables for the skewed quark distributions, $\widetilde{H}_{q}\left(\tau, \xi, \Delta^{2}\right)$ and $\widetilde{E}_{q}\left(\tau, \xi, \Delta^{2}\right)$, the variable $\tau$, related to the fraction of target momentum carried by the interacting parton $x_{1}$ by:

$$
\tau=\frac{x_{1}-\frac{x}{2}}{1-\frac{x}{2}}
$$

the square of the four-momentum transfer, $\Delta^{2}=t$, and the light-cone fraction of $\Delta-\xi$ (the skewedness parameter) p?.

In the forward case, $p=p^{\prime}$, both $\Delta$ and $\xi$ are zero, and the second term on the r.h.s. of eq.(20) disappears. The function $\widetilde{H}_{q}$ becomes the usual polarized parton distribution

\footnotetext{
${ }^{3}$ The skewed parton distributions and meson distribution amplitudes are scale dependent; the scale is set by the photon virtuality $Q^{2}$. We do not show the scale dependence of these quantities so as to simplify the notation.
} 
function,

$$
\widetilde{H}_{q}(\tau, \xi=0, t=0)=\Delta q(\tau)=\left\{\begin{array}{cl}
\Delta q(\tau)=q_{+}(\tau)-q_{-}(\tau), & \tau>0 \\
\Delta \bar{q}(-\tau)=\bar{q}_{+}(-\tau)-\bar{q}_{-}(-\tau), & \tau<0
\end{array}\right.
$$

Here $q_{ \pm}(\tau)$ are densities of partons with positive (negative) helicity in the proton with positive longitudinal polarization. Let us note that the universal function $\widetilde{H}_{q}(\tau, \xi=$ $0, t=0$ ) corresponds to the sum of valence and sea polarized quark distributions. At the same time only the valence polarized quark distributions give nonzero contribution to the amplitudes of the electroproduction of neutral pseudoscalar mesons. We want to draw attention that these distributions are not constrained by inclusive DIS experiments. This is because of the negative charge parity in the $t$-channel for the amplitude for electroproduction of electrically neutral pseudoscalar meson. However these distributions are probed in semiinclusive DIS experiments.

It is useful to define isoscalar:

$$
\begin{aligned}
\widetilde{H}^{(0)}(\tau, \xi, t) & =\widetilde{H}_{u}(\tau, \xi, t)+\widetilde{H}_{d}(\tau, \xi, t), \\
\widetilde{E}^{(0)}(\tau, \xi, t) & =\widetilde{E}_{u}(\tau, \xi, t)+\widetilde{E}_{d}(\tau, \xi, t),
\end{aligned}
$$

and isovector

$$
\begin{aligned}
\widetilde{H}^{(3)}(\tau, \xi, t) & =\widetilde{H}_{u}(\tau, \xi, t)-\widetilde{H}_{d}(\tau, \xi, t), \\
\widetilde{E}^{(3)}(\tau, \xi, t) & =\widetilde{E}_{u}(\tau, \xi, t)-\widetilde{E}_{d}(\tau, \xi, t),
\end{aligned}
$$

skewed quark distributions.

The SPD's $\widetilde{H}^{(3)}(\tau, \xi, t)$ and $\widetilde{E}^{(3)}(\tau, \xi, t)$ satisfy the sum rules [6]:

$$
\begin{aligned}
\int_{-1}^{1} d \tau \widetilde{H}^{(3)}(\tau, \xi, t) & =G_{A}(t), \\
\int_{-1}^{1} d \tau \widetilde{E}^{(3)}(\tau, \xi, t) & =G_{P}(t),
\end{aligned}
$$

where $G_{A}(t)\left(G_{A}(0)=g_{A} \approx 1.25\right)$ and $G_{P}(t)$ are the axial and pseudoscalar form factors of the nucleon.

In the next sections we will consider polarization effects in the production of pseudoscalar mesons off a polarized proton. To be specific we will discuss pion electroproduction. All formulae can be easily generalized to the case of production of other pseudoscalar mesons using the general expression for the production amplitude (丑) and isospin $\left(S U_{f l}(3)\right)$ relations (15,17).

For the charged pion production the leading order amplitude can be written as:

$$
\begin{aligned}
& \left\langle n\left(p^{\prime}\right), \pi^{+}\left(p+q-p^{\prime}\right)\left|J^{\text {e.m. }} \cdot \varepsilon_{L}\right| p(p)\right\rangle=-\left(i e 4 \pi \alpha_{s}\right) \frac{N_{c}^{2}-1}{N_{c}^{2}} \frac{1}{24 Q} f_{\pi} \int_{0}^{1} d z \frac{\varphi_{\pi}(z)}{z} \\
& \times\left\{\bar{U}\left(p^{\prime}\right) \hat{n} \gamma_{5} U(p) \int_{-1}^{1} d \tau \widetilde{H}^{(3)}(\tau, \xi, t)\left(3 \alpha^{-}(\tau)-\alpha^{+}(\tau)\right)\right. \\
& \left.+\bar{U}\left(p^{\prime}\right) \frac{n \cdot \Delta}{2 M_{N}} \gamma_{5} U(p) \int_{-1}^{1} d \tau \widetilde{E}^{(3)}(\tau, \xi, t)\left(3 \alpha^{-}(\tau)-\alpha^{+}(\tau)\right)\right\},
\end{aligned}
$$


where

$$
\alpha^{ \pm}(\tau)=\frac{1}{\tau+\frac{\xi}{2}-i 0} \pm \frac{1}{\tau-\frac{\xi}{2}+i 0},
$$

is the hard kernel computed in the leading order of $\alpha_{s}$.

The neutral pion production amplitude has the form (this amplitude was obtained previously in [11, 12 |7):

$$
\begin{aligned}
& \left\langle p\left(p^{\prime}\right), \pi^{0}\left(p+q-p^{\prime}\right)\left|J^{\text {e.m. }} \cdot \varepsilon_{L}\right| p(p)\right\rangle=-\left(i e 4 \pi \alpha_{s}\right) \frac{N_{c}^{2}-1}{N_{c}^{2}} \frac{1}{24 \sqrt{2} Q} f_{\pi} \int_{0}^{1} d z \frac{\varphi_{\pi}(z)}{z} \\
& \times\left\{\bar{U}\left(p^{\prime}\right) \hat{n} \gamma_{5} U(p) \int_{-1}^{1} d \tau\left(\widetilde{H}^{(3)}(\tau, \xi, t)+3 \widetilde{H}^{(0)}(\tau, \xi, t)\right) \alpha^{+}(\tau)\right. \\
& \left.+\bar{U}\left(p^{\prime}\right) \frac{n \cdot \Delta}{2 M_{N}} \gamma_{5} U(p) \int_{-1}^{1} d \tau\left(\widetilde{E}^{(3)}(\tau, \xi, t)+3 \widetilde{E}^{(0)}(\tau, \xi, t)\right) \alpha^{+}(\tau)\right\} .
\end{aligned}
$$

Let us illustrate at the end of this section the possible applications of the $S U_{f l}(3)$ relations eqs. (15, 17, 18). With their help we can roughly estimate the ratios of yields of kaons to pions in hard exclusive electroproduction reactions. For instance, we can easily estimate the ratio of yields of $\pi^{+}(n)$ and $K^{+}(\Lambda)$ at small $x\left(x<m_{\pi} / M_{N}\right)$ f as:

$$
\frac{K^{+}}{\pi^{+}} \approx \frac{f_{K}^{2}}{6 f_{\pi}^{2}} \frac{\left[3\left(2 \Delta u_{s}-\Delta d_{s}-\Delta s_{s}\right)-\left(2 \Delta u_{v}-\Delta d_{v}-\Delta s_{v}\right)\right]^{2}}{\left[3\left(\Delta u_{s}-\Delta d_{s}\right)-\left(\Delta u_{v}-\Delta d_{v}\right)\right]^{2}} .
$$

Here we introduce valence $\Delta q_{v}=\Delta q-\Delta \bar{q}$ and sea $\Delta q_{s}=\Delta q+\Delta \bar{q}$ polarized quark distributions. These quantities are antisymmetric and symmetric under transposition $s \leftrightarrow u$ and have signatures -1 and 1 . (Note that our definition of $\Delta q_{s}$ differes from the often used definition $\Delta q_{s}=2 \Delta \bar{q}$.) We want to draw attention that in this case both the sea quark $\left(\mathbf{8}_{\mathbf{F}}\right)$ and valence quarks $\left(\mathbf{8}_{\mathbf{D}}\right)$ contribute to the amplitude, see eq.(17).

For the ratio of $K^{0}$ and $\pi^{0}$ yields we get the estimate:

$$
K^{0}: \pi^{0} \approx 2 f_{K}^{2}\left(\Delta d_{v}-\Delta s_{v}\right)^{2}: f_{\pi}^{2}\left(2 \Delta u_{v}+\Delta d_{v}\right)^{2} \approx 3: 1
$$

where we take for simplicity $\Delta s_{v}=\Delta u_{v}+\Delta d_{v} \approx 0$ for $x=0.1 \div 0.4$ (this is also in line with large $N_{c}$ counting, see below). Surprisingly large ratios!

\section{Spin asymmetry}

The expressions for the amplitudes (26,28) contain the full information about the reaction $\gamma_{L}^{*}+p \rightarrow \pi^{a}+N$ in the leading order of $1 / Q^{2}$ expansion. In the present paper we consider a specific polarization observable, for which we expect (see the discussion in the introduction) a precocious scaling at moderately large $Q^{2} \sim 2 \div 4 \mathrm{GeV}^{2}$. We expect that

\footnotetext{
${ }^{4}$ Ref. [11] used a different spin decomposition of SPD's from those here.

${ }^{5}$ For larger $x$ we have to take into account large contributions of $\pi^{ \pm}$and $K^{+}, K^{0}$ poles to the spin flip SPD $\widetilde{E}$ (see discussion below).
} 
for the absolute cross section the onset of scaling occurs at larger values of $Q^{2} \geq 10 \mathrm{GeV}^{2}$. Also the spin asymmetry is the most sensitive observable to probe the spin-flip SPD $\widetilde{E}$.

Let us consider production of the pion off a polarized proton. The differential cross section can be written in the form:

$$
\sigma=\sigma_{0}+\sigma_{1}\left(\left[\vec{p}_{\perp}^{\prime}, \vec{S}_{\perp}\right] \cdot \vec{e}_{z}\right) /\left|\vec{p}_{\perp}^{\prime}\right|=\sigma_{0}+\sigma_{1}\left|\vec{S}_{\perp}\right| \sin \beta
$$

where $S_{\perp}$ is the transverse projection of the polarization vector of the initial proton (or of the polarization vector of the outgoing nucleon taken with "-" sign), $\vec{e}_{z}$ is a vector normal to this plane and $\beta$ is the angle between the $S_{\perp}$ and $p_{\perp}^{\prime}$ (transverse component of the momentum of the outgoing nucleon).

Using the above expressions for the leading twist amplitudes of the charged (26) and neutral (28) pion production we define:

$$
\begin{aligned}
& A_{+}=\int_{-1}^{1} d \tau \widetilde{H}^{(3)}(\tau, \xi, t)\left(3 \alpha^{-}(\tau)-\alpha^{+}(\tau)\right) \\
& B_{+}=\int_{-1}^{1} d \tau \widetilde{E}^{(3)}(\tau, \xi, t)\left(3 \alpha^{-}(\tau)-\alpha^{+}(\tau)\right),
\end{aligned}
$$

for the charged pion production, and

$$
\begin{aligned}
& A_{0}=\int_{-1}^{1} d \tau\left(\widetilde{H}^{(3)}(\tau, \xi, t)+3 \widetilde{H}^{(0)}(\tau, \xi, t)\right) \alpha^{+}(\tau) \\
& B_{0}=\int_{-1}^{1} d \tau\left(\widetilde{E}^{(3)}(\tau, \xi, t)+3 \widetilde{E}^{(0)}(\tau, \xi, t)\right) \alpha^{+}(\tau),
\end{aligned}
$$

for the neutral pion production. For definitness let us consider the following asymmetry:

$$
\mathcal{A}=\frac{1}{\left|S_{\perp}\right|} \frac{\int_{0}^{\pi} d \beta|\mathcal{M}(\beta)|^{2}-\int_{\pi}^{2 \pi} d \beta|\mathcal{M}(\beta)|^{2}}{\int_{0}^{2 \pi} d \beta|\mathcal{M}(\beta)|^{2}}=\frac{2 \sigma_{1}}{\pi \sigma_{0}} .
$$

It follows from our definition of the kinematical variables (9) that $p_{\perp}^{\prime}=\Delta_{\perp} / 2$ with $\Delta_{\perp}^{2}=-\frac{4(1-x)}{(2-x)^{2}}\left(t-t_{\min }\right)+O\left(1 / Q^{2}\right)$, where $t_{\min }=-M_{N}^{2} x^{2} /(1-x)+O\left(1 / Q^{2}\right)$ is the minimal (in absolute value) momentum transfer squared in the reaction. In terms of SPD's the asymmetry $\mathcal{A}$ has the form (for the charged and neutral pion production):

$$
\mathcal{A}_{+, 0}=\frac{\left|\Delta_{\perp}\right|}{\pi M_{N}} \frac{\xi \operatorname{Im}\left(A_{+, 0} B_{+, 0}^{*}\right)}{\left|A_{+, 0}\right|^{2}\left(1-\frac{\xi^{2}}{4}\right)-\left|B_{+, 0}\right|^{2} \frac{t \xi^{2}}{16 M_{N}^{2}}-\frac{\xi^{2}}{2} \operatorname{Re}\left(A_{+, 0} B_{+, 0}^{*}\right)} .
$$

From this expression we see immediately that the asymmetry $\mathcal{A}$ would be zero if the spin flip SPD $\widetilde{E}$ were zero. In contrast to the $\operatorname{SPD} \widetilde{H}_{q}(\tau, \xi, t)$ which in the forward limit $t \rightarrow 0$ reduces to the usual polarized quark distributions $\widetilde{H}(\tau, \xi=0, t=0)=\Delta q(\tau)$, the SPD $\widetilde{E}(\tau, \xi, t)$ can not be related to already known quark distributions. For the estimates of the asymmetry (35) we shall resort to the computation of the skewed parton distributions in the large $N_{c}$ limit in the chiral quark-soliton model [20, 19]. 


\section{Skewed parton distributions in the large $N_{c}$ limit and spin asymmetry}

Recently the skewed quark distribution $\widetilde{H}$ and $\widetilde{E}$ were computed in the chiral quarksoliton model of the nucleon [19]. In this model nucleons can be viewed as $N_{c}$ "valence" quarks bound by a self-consistent pion field (the "soliton") whose energy coincides with the aggregate energy of the quarks of the negative-energy Dirac continuum. Similarly to the Skyrme model, the large $N_{c}$ limit is needed to justify the use of the mean-field approximation.

In 21] a new approach to the calculation of quark distribution functions has been developed within the context of the chiral quark-soliton model of the nucleon [22], and furthermore in [20] this method was applied to calculate SPD's. The range of the applicability of the chiral quark-soliton model to the parton distributions is limited by the conditions: $|t| \ll M_{N}^{2}, \tau, \xi \sim 1 / N_{c}$, the computed distributions refer to a low normalization point of about $600 \mathrm{MeV}$.

According to the large $N_{c}$ analysis of ref. [19] $\widetilde{H}^{(0)} \ll \widetilde{H}^{(3)}$ and $\widetilde{E}^{(0)} \ll \widetilde{E}^{(3)}$ in the large $N_{c}$ limit which is in line with the phenomenology for the diagonal case. Another crucial observation made in [19] is that the isovector skewed quark distribution $\widetilde{E}^{(3)}$ has a strong enhancement at small $t$ originating from the long range pion tail of the nucleon wave function.

This contribution has the form [19]:

$$
\widetilde{E}_{\pi}^{(3)}=\theta\left[|\tau|<\frac{|\xi|}{2}\right] \Phi_{\pi}\left(\frac{2 \tau}{\xi}\right) \frac{2 F(t)}{\xi},
$$

where $\Phi_{\pi}(z)$ is the pion distribution amplitude, normalized by $\int_{-1}^{1} d z \Phi_{\pi}(z)=1\left(\Phi_{\pi}(z)=\right.$ $\left.\frac{1}{2} \varphi_{\pi}\left(\frac{1-z}{2}\right)\right)$. The pion distribution amplitude $\Phi_{\pi}(x)$ in eq. (39) calculated in the same model [23, 24] is very close to the asymptotic one $\Phi_{\pi}(z)=\frac{3}{4}\left(1-z^{2}\right)$, so we shall always use the asymptotic pion distribution amplitude for numerical calculations. The form factor $F(t)$ in this model is related to the Fourier transform of the mean pion field $U(x)=\exp \left(i \pi^{a}(x) \tau^{a}\right)$ :

$$
F\left(-\vec{k}^{2}\right)=\frac{2 M_{N}^{2} f_{\pi}^{2}}{3 k^{3}} \int d^{3} x \exp (i \vec{k} \cdot \vec{x}) \operatorname{Tr}\left[(U(x)-1) \tau^{3}\right]
$$

Taking into account that at large distances the soliton field has the Yukawa tail (see e.g. [22]):

$$
\lim _{r \rightarrow \infty}(U(x)-1)=\frac{i x^{a} \tau^{a}}{r} \frac{3 g_{A}}{4 \pi f_{\pi}^{2} r^{2}}\left(1+m_{\pi} r\right) \exp \left(-m_{\pi} r\right)
$$

one gets immediately the small $t$ asymptote of the form factor $F(t)$ :

$$
\lim _{t \rightarrow m_{\pi}^{2}} F(t)=-\frac{4 g_{A} M_{N}^{2}}{t-m_{\pi}^{2}} .
$$


The numerical results for the form factor $F(t)$ can be found in [19]. Let us note that the form factor $F(t)$ in a wide range of $t: m_{\pi}^{2} \ll|t| \ll M_{N}^{2}$ differs significantly from the pole contribution (39). For example, for $-t=0.35 \mathrm{GeV}^{2}$ they differ by a factor of two. In our numerical estimates of the asymmetry we shall use the results of 19.

The pion pole contribution (39) corresponds to the pion pole contribution to the nucleon form factor $G_{P}(t)$ |):

$$
\lim _{t \rightarrow m_{\pi}^{2}} \int_{-1}^{1} d \tau \widetilde{E}_{\pi}^{(3)}(\tau, \xi, t)=-\frac{4 g_{A} M_{N}^{2}}{t-m_{\pi}^{2}}
$$

required by spontaneously broken chiral symmetry. We see that the appearance of the pion pole in $\widetilde{E}^{(3)}$ is a general consequence of PCAC and in order to reproduce it in some model it should respect the chiral Ward identities. For example, in the computation of SPD's in the bag model 25] the chiral Ward identities are violated and the pion pole contribution (39) is missed. The chiral quark-soliton model respects all chiral Ward identities that allows one to split unambiguously SPD $\widetilde{E}^{(3)}$ into two pieces 19:

$$
\widetilde{E}^{(3)}(\tau, \xi, t)=\widetilde{E}_{\pi}^{(3)}(\tau, \xi, t)+\widetilde{E}_{\text {smooth }}^{(3)}(\tau, \xi, t),
$$

the result for $\widetilde{E}_{\pi}^{(3)}$ is given by eq. (36); the results for $\widetilde{E}_{\mathrm{smooth}}^{(3)}(\tau, \xi, t)$ can be found in 19].

It is easy to see that $\widetilde{E}_{\pi}^{(3)}(\tau, \xi, t)$ does not contribute to the $B_{0}$ amplitude of the neutral pion production (see eqs. (33)). However, it contributes to the $B_{+}$amplitude of the charged pion production (see eqs. (32)). The corresponding contribution has the form:

$$
B_{+}^{(\pi)}=\frac{12 F(t)}{\xi} \int_{-1}^{1} d z \frac{\Phi_{\pi}(z)}{1-z^{2}}
$$

We see that the SPD $\widetilde{E}_{\pi}^{(3)}$ eq.(36) contributes only to the real part of amplitude $B_{+}$, also it is strongly enhanced at small $t$ and $\xi$. Using the results of ref. [19] we obtain that $\operatorname{Re} B_{+}^{\pi} \gg \operatorname{Re} B_{+}^{\text {smooth }}, \operatorname{Im} B_{+}$at least by an order of magnitude for $|t|<0.5 \mathrm{GeV}^{2}$ and $0.1<x<0.4$. In the case of the neutral pion production with the spin flip amplitude $B_{0}$, the pion pole does not show up. As a result $B_{0}$ and $B_{+}^{\text {smooth }}$ should have the same magnitude. This result immediately implies that

$$
\mathcal{A}_{0} \ll \mathcal{A}_{+}
$$

Neglecting $\operatorname{Re} B_{+}^{\text {smooth }}$ and $\operatorname{Im} B_{+}$relative to $B_{+}^{(\pi)}$ we get the following expression for the asymmetry (35) $\mathcal{A}_{+}$of charged pion production

$$
\mathcal{A}_{+} \approx \frac{\left|\Delta_{\perp}\right|}{\pi M_{N}} \frac{18 \eta F(t) \operatorname{Im} A_{+}}{\left|A_{+}\right|^{2}\left(1-\frac{\xi^{2}}{4}\right)-\frac{81 \eta^{2} t}{4 M_{N}^{2}} F(t)^{2}-9 \xi F(t) \operatorname{Re} A_{+}},
$$

where $\eta=\frac{2}{3} \int_{-1}^{1} d z \frac{\Phi_{\pi}(z)}{1-z^{2}}(\eta=1$ for the asymptotic pion distribution amplitude).

\footnotetext{
${ }^{6} \mathrm{~A}$ similar expression is valid for the contribution of the kaon pole.
} 
In Fig. 1 we plot the asymmetry (44) at several values of $t$ as a function of $x$. We plot the asymmetry for $\eta=1$ (corresponding to the asymptotic pion distribution amplitude). We show also the result for $\eta=5 / 3$ (corresponding to Chernyak-Zhitnitsky model for the pion DA) merely to illustrate the sensitivity of the asymmetry to the shape of the pion distribution amplitude. The instanton model gives the almost asymptotic pion distribution amplitude (see [23, 24]) already at a low normalization point of about $600 \mathrm{MeV}$. One can see that the spin asymmetry in the production of charged pions is of order unity, this makes challenging the measurements of this asymmetry. Alternatively, one can measure the recoil nucleon polarization. Also it is seen that the sensitivity to the form of the pion distribution amplitude is maximal at small $t$.

Let us analyze eq. (44) at small momentum transfer $|t|<m_{\pi}^{2}$ and small Bjorken $x<m_{\pi} / M_{N}$. In this range of $t$ and $x$ the contribution of the pion pole (the spin flip part of the amplitude) to the absolute cross section is suppressed, whereas for the spin asymmetry we have the following expression:

$$
\begin{aligned}
\mathcal{A}_{+} & \approx \frac{72\left|\Delta_{\perp}\right| \eta g_{A} M_{N}}{\pi m_{\pi}^{2}} \frac{\operatorname{Im} A_{+}}{\left|A_{+}\right|^{2}} \\
& =\frac{144\left|\Delta_{\perp}\right| \eta g_{A} M_{N}}{m_{\pi}^{2}} \frac{\left(2 \widetilde{H}^{(3)}\left(\frac{\xi}{2}, \xi, t\right)+\widetilde{H}^{(3)}\left(-\frac{\xi}{2}, \xi, t\right)\right)}{\left|A_{+}\right|^{2}} .
\end{aligned}
$$

We see that the asymmetry in the electroproduction of charged pions in this limit depends on both the polarized valence and sea quark distributions. The particular combination which enters in this case is poorly constrained by the DIS measurements. This makes the hard exclusive pion production experiments a useful source of information on the polarized quark distributions.?

\section{$5 \quad$ Hard Coherent production of pions off nuclei}

In this section we discuss briefly the hard exclusive production of pseudoscalar mesons off nuclei. We focus primarily on the polarization effects and comment also on the peculiarities of the $A$-dependence related to the spin structure of the elementary amplitude.

First let us consider the scattering off deuteron targets. We explained above that the amplitude for the electroproduction of a neutral pseudoscalar meson (28) (in contrast to the charged pion production amplitude) is dominated at small $t$ by the helicity nonflip amplitude $A_{0}$. In the (near) forward limit $A_{0}$ is proportional to the spin of nucleon - $\phi\left(p^{\prime}\right) \sigma_{z} \phi(p)$, where $z$ is the photon momentum direction and $\phi(p)$ is the spin part of the wave function of the nucleon. Therefore, in the case of the coherent scattering off a polarized deuteron target at small $t$ the dominant term is proportional to the projection of the sum of the operators of the nucleon spins to the reaction axis with the proportionality coefficient calculable in terms of the isoscalar polarized valence quark distributions. If this

\footnotetext{
${ }^{7}$ Note that if the process $e+p \rightarrow e+\pi^{+}+n$ is studied via a setup detecting only the scattered electron and $\pi^{+}$one may not have good enough energy resolution to separate this process from the process $e+p \rightarrow e+\pi^{+}+\Delta^{0}$. However our estimates using the methods outlined in [18] indicate that this would result only in a slight dilution of the asymmetry.
} 
projection is zero, the corresponding amplitude should be zero for the forward scattering. This is because the matrix element of the projection of the sum of spins of nucleons is equal to zero even if the $D$-wave in the deuteron wave function is taken into account. In the case of the charged pion production dominance of the spin-flip amplitude leads to a strong excitation of the isotriplet two-nucleon virtual state.

Meson production off heavier nuclei in the discussed limit constitutes another class of color coherent phenomena. Since the mesons are produced in small size configurations they do not interact with the residual system within the leading twist approximation. Hence the amplitudes of such processes are expressed through the skewed parton density of the nucleus and can be represented (neglecting small EMC like effects) as a convolution of the elementary amplitude and the nuclear transition amplitude:

$$
M(\Delta)=\int d^{3} \vec{k}\left\langle A^{\prime}(\vec{\Delta})\left|\delta\left(H_{A^{\prime}}-\Delta_{0}\right) a_{s^{\prime}, N_{f}}^{\dagger}(\vec{k}+\vec{\Delta}) a_{s, N_{i}}(\vec{k})\right| A\right\rangle,
$$

where $\Delta$ is the four-momentum transfer in the nucleus rest frame, and $a^{\dagger}, a$ are production and annihilation operators for nucleons with given spin states. A correlation between these spin states is implicitly contained in the elementary amplitude. Such matrix elements can be calculated using conventional methods of low-energy nuclear physics. For large enough $t$ one can use closure over the processes of nuclear disintegration $A^{\prime}$ and write (we give the answer for production of neutral mesons):

$$
\sum_{A^{\prime}} \frac{d \sigma\left(\gamma_{L}^{*}+A \rightarrow M+A^{\prime}\right)}{d t}=Z \frac{d \sigma\left(\gamma_{L}^{*}+p \rightarrow M+p^{\prime}\right)}{d t}+N \frac{d \sigma\left(\gamma_{L}^{*}+n \rightarrow M+n^{\prime}\right)}{d t},
$$

where $Z$ and $N$ are the numbers of protons and neutrons in the target.

For small enough $t$ the result is strongly sensitive to the spin effects. Let us give several examples.

(a) For the case of $\pi^{0}$ production off ${ }^{3} \mathrm{He}$ one finds for small $t$ a strong cancelation of the contribution of the scattering off the protons (in the approximation that ${ }^{3} \mathrm{He}$ is described as a $S$-wave system).

$$
\frac{d \sigma\left(\gamma_{L}^{*}+{ }^{3} H e \rightarrow \pi^{0}+{ }^{3} H e\right)}{d t}=\frac{d \sigma\left(\gamma_{L}^{*}+n \rightarrow \pi^{0}+n\right)}{d t} F_{A=3}(t),
$$

where $F_{A=3}(t)$ is a superposition of the magnetic and electric ${ }^{3} \mathrm{He}$ form factors normalized to one at $t=0$.

(b) for reaction $\gamma_{L}^{*}+{ }^{3} H e \rightarrow \pi^{+}+{ }^{3} H$ for small $t$ one expects spin asymmetries similar to the case of scattering off a proton which we discussed above. If, in line with our expectations, the spin-flip amplitude dominates in the elementary cross section for small $t$, the cross section will be approximately equal to

$$
\frac{d \sigma\left(\gamma_{L}^{*}+{ }^{3} H e \rightarrow \pi^{+}+{ }^{3} H\right)}{d t}=\frac{d \sigma\left(\gamma_{L}^{*}+p \rightarrow \pi^{+}+n\right)}{d t} F_{M, A=3}(t)
$$

where $F_{M, A=3}(t)$ is the magnetic form factor of ${ }^{3} \mathrm{He}$ normalized to one at $t=0$.

(c) In the case of $\pi^{0}$ production off ${ }^{4} \mathrm{He}$ for small $t$ we expect a much stronger suppression of the cross section than in the case of scattering off the deuteron. Indeed, in 
this case the dominant contribution in the elementary amplitude is a vector in the spin indices which cancels out when averaged with the $|i n\rangle$ and $\langle$ out $|$ spin zero states of ${ }^{4} \mathrm{He}$.

The $K^{+}$meson production is also of interact for the study of the low-energy hyperonnucleus interactions. In a sense, we have here a situation of a local "micro" implantation of a hyperon with a small momentum in any point in the nucleus, predominantly in the central region, without disturbing any other nucleons. This is markedly different from the pion induced processes where for heavy nuclei only production near the surface is important while the final state interactions of the outgoing kaon cannot be neglected.

To summarise, studies of the scattering off nuclei will provide new handles for the study of spin effects, effects of color coherence, as well as of the spin structure of low-energy interactions.

\section{Exclusive production of forward baryons off nucle- ons and meson implantation in nuclei}

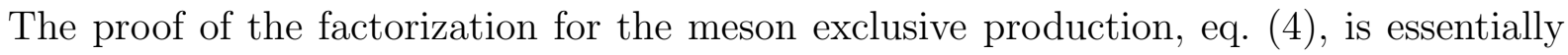
based on the observation that the cancellation of the soft gluon interactions is intimately related to the fact that the meson arises from a quark-antiquark pair generated by the hard scattering. Thus the pair starts as a small-size configuration and only substantially later grows to a normal hadronic size, to a meson. This implies that the parton density is a standard parton density (apart from the skewed nature of its definition). Therefore the factorization theorem is valid also for the production of leading baryons

$$
\gamma^{*}(q)+p \rightarrow B(q+\Delta)+M(p-\Delta)
$$

and even leading antibaryons

$$
\gamma^{*}(q)+p \rightarrow \bar{B}(q+\Delta)+B_{2}(p-\Delta),
$$

where $B_{2}$ is a system with the baryon charge of two. Processes (50,51) will provide unique information about multiparton correlations in nucleons. For example, process (50) will allow one to investigate the probability for three quarks in a nucleon to come close together without collapsing the wave function into a three quark component - such a probability would not be small in meson cloud models of the nucleon and in, say, the MIT bag model. On the other hand if one would try to follow an analogy with the case of the positronium, this probability would be strongly suppressed. At the same time reaction (51) would allow one to determine a probability (presumably numerically very small) to have three antiquarks close together in a nucleon.

To describe in QCD process (50), a new non-perturbative mathematical object should be introduced in addition to the usual distribution amplitudes (DA). It can be called a skewed distribution amplitude (SDA.) It is defined as a non-diagonal matrix element of the tri-local quark operator between a meson $M$ and a proton:

$$
\begin{aligned}
& \left.\int \prod_{i=1}^{3} d z_{i}^{-} \exp \left[i \sum_{i=1}^{3} x_{i}\left(p \cdot z_{i}\right)\right]\left\langle M(p-\Delta)\left|\varepsilon_{a b c} \psi_{j_{1}}^{a}\left(z_{1}\right) \psi_{j_{2}}^{b}\left(z_{2}\right) \psi_{j_{3}}^{c}\left(z_{3}\right)\right| N(p)\right\rangle\right|_{z_{i}^{+}=z_{i}^{\perp}=0} \\
& =\delta\left(1-\zeta-x_{1}-x_{2}-x_{3}\right) F_{j_{1} j_{2} j_{3}}\left(x_{1}, x_{2}, x_{3}, \zeta, t\right)
\end{aligned}
$$


where $a, b, c$ are color indices, $j_{i}$ are spin-flavor indices, and $F_{j_{1} j_{2} j_{3}}\left(x_{1}, x_{2}, x_{3}, \zeta, t\right)$ are the new SDA. These SDA can be decomposed into invariant spin-flavor structures which depend on the quantum numbers of the meson $M$. They depend on the variables $x_{i}$ (which are contracted with the hard kernel in the amplitude), on the skewedness parameter $\zeta=1-\Delta^{+} / p^{+}$(in some sense, with this definition of $\zeta$ the limit $\zeta \rightarrow 0$ corresponds to the usual distribution amplitude, i.e. skewedness $\rightarrow 0$ means SDA $\rightarrow$ DA) and the momentum transfer squared $t=\Delta^{2}$.

Though quantitative calculations of processes (50, 51) will take time, some qualitative predictions could be made right away: the cross section of the process for fixed $x$, and large $Q^{2}$ should be proportional to the baryon elastic form factor. In particular, it would be instructive to study the $Q^{2}$ dependence of the ratio of the cross section for the process $\gamma^{*}(q)+p \rightarrow p+\pi^{0}$ and the square of the elastic proton form factor. If the color transparency suppresses the final state interaction between the fast moving nucleon and the residual meson state early enough one may expect that this ratio may reach the scaling limit in the region where higher twist contributions to the nucleon form factor are still large. (It is worth emphasizing that the longitudinal distances involved in the final state interaction of the system flying along $\vec{q}$ with the residual system are much smaller in this case than in the case of the $A\left(e, e^{\prime} p\right)$ reaction, so the expansion effects would be much less important in this case.) Another interesting process is $\gamma^{*}(q)+p \rightarrow \Delta^{++}+\pi^{-}$which may allow one to compare the wave functions of the $\Delta$-isobar and a nucleon in a way complementary to the $N \rightarrow \Delta$ transition processes. Note also, that a study of the meson spectrum in the process $\gamma^{*}+p \rightarrow p+M$ may help to investigate the role of pions in the nucleon wave function. In a naive model of the nucleon with a pion cloud one would expect that the single pion production will dominate. However if more complicated nonlinear pion fields are important in the low $Q^{2} q \bar{q}$ sea, one may expect that higher recoil masses would be at least as important.

Reaction (50) provides also a promising avenue to look for exotic meson states including gluonium. Indeed, if one would consider, for example, the MIT bag model, the removal of three quarks from the system could leave the residual system looking asw like a bag made predominantly of glue. It is natural to expect that such a system would have a large overlapping integral with gluonium states.

An interesting property of reaction (50) is that it could be used to produce a meson " $M "(\eta, \rho, K$-meson with zero momentum in the nucleon target rest frame) provided

$$
q_{0}=\frac{Q^{2}+2 m_{M} m_{N}-m_{M}^{2}}{2\left(m_{N}-m_{M}\right)}
$$

In the case of the production of a leading baryon off the nuclear target, similar to the case of the production of leading mesons discussed in section 5, the outgoing nucleon would not interact with the nucleus since it is produced in a small-size configuration. Hence it is possible in the discussed reactions to "implant" a meson with a small momentum in the center of the nucleus. This implantation has a number of advantages as compared to the processes of low-energy implantation since in the low energy processes mesons are produced predominantly near the surface and the interaction of other hadrons involved in the process is, in general, large. Therefore process (50) could be used for studying the medium modification of the properties of various mesons. In the case of $\rho$-meson 
production an additional advantage of this process as compared to low-energy processes is that in the high-energy case the effects of distortion of the two pion mass spectrum due threshold effects are much less important.

\section{Conclusions}

We have derived the general expression for the hard pseudoscalar meson $(\pi, K$, etc.) electroproduction amplitude in QCD in the leading order in $1 / Q^{2}$ and $\alpha_{s}$.

We have demonstrated that the distribution of the recoil nucleons in the hard exclusive production of $\pi^{+}$strongly depends on the angle between the momentum of the recoil nucleon and the spin polarization of the target (or the outgoing nucleon). This dependence is especially sensitive to the helicity flip skewed parton distribution (SPD) $\widetilde{E}$. This helicity flip SPD contains at small momentum transfer the contribution of the pion pole due to the chiral Ward identities. Owing to the factorization theorem for the hard exclusive processes (1] the relative size of pole and non-pole contributions can be estimated quantitatively using the low-energy chiral model of the nucleon [19.

For $x \rightarrow 0$ (i.e. $t=t_{\min } \rightarrow 0$ ) hard electroproduction of charged mesons gives a unique possibility to measure the $x$ dependence of the polarized valence quark distribution in a nucleon (nuclear) target. This is because skewed parton distributions are calculable in this limit in terms of the conventional valence quark distributions in a target. Such an investigation is especially interesting since in this limit this $x$ dependence should be different from the one for the unpolarized case due to different quantum numbers in the crossed channel. Additionally, comparing reactions with different flavor quantum numbers, like e.g.:

$$
\begin{aligned}
& \gamma_{L}^{*}+p \rightarrow \pi^{+}+n, \\
& \gamma_{L}^{*}+p \rightarrow K^{+}+\Lambda, \\
& \gamma_{L}^{*}+p \rightarrow K^{+}+\Sigma^{0} \\
& \gamma_{L}^{*}+p \rightarrow K^{0}+\Sigma^{+},
\end{aligned}
$$

one can access different flavor combinations of polarized quark distributions in the proton, and probe $S U_{f l}(3)$ for spin part of the strange baryon quark densities (see eqs. (15, 17, 18)).

We have also demonstrated that the study of exclusive meson production off the nuclear target will provide addition information about the spin structure of skewed distributions as well as allow one to look for color coherence effects.

\section{Acknowledgments}

We would like to acknowledge discussions with K. Goeke, L. Mankiewicz, G. Piller, A. Radyuskin, A. Shuvaev, M. Vanderhaeghen and C. Weiss. We thank I. Börnig and M. Penttinen for help with numerical calculations. We thank J.Whitmore for reading the paper and useful comments. The work of P.V.P. and M.V.P. has been supported by RFBR grant 96-15-96764 and the joint grant of RFBR and the Deutsche Forschungsgemeinschaft (DFG) 436 RUS 113/181/0 (R), by the BMBF grant RUS-658-97 and the COSY (Jülich). 
M.S. would like to thank DESY for hospitality during the time this work was done. The work of M.S. and L.F. is supported in part by the U.S. Department of Energy and BSF. After completing the paper we learned that the cross section of $\pi^{+}$hard electroproduction was calculated independently by L. Mankiewicz, G. Piller and A. Radyushkin [26]. We are grateful to them for the discussions and an exchange of results.

\section{References}

[1] J. Collins,L. Frankfurt, and M. Strikman, Phys. Rev. D56 (1997) 2982.

[2] J. Bartels and M. Loewe, Z.Phys. C12 (1982) 263

[3] I.I. Balitskii, V.M. Braun, Nucl.Phys. B311 (1989) 541.

[4] F.M. Dittes, D. Muller, D. Robaschik, B. Geyer, J. Horejsi Phys.Lett. B209 (1988) 325.

[5] H. Abramowicz, L.L.Frankfurt, and M.Strikman, DESY preprint 95-047,Proceedings of SLAC 94 Summer School SLAC Report 484,pp 539-574, Survey High Energy Physics 11 (1997) 51, hep-ph/9503437

[6] X. Ji, Phys. Rev. Lett. 78 (1997) 610; X. Ji, Phys. Rev.D55 (1997) 7114.

[7] A. V. Radyushkin, Phys. Lett. B380 (1996) 417.; A. V. Radyushkin, Phys. Rev. D56 (1997)

[8] X. Ji, J. Phys. G24 (1998) 1181.

[9] L.L. Frankfurt et al, Phys.Lett.B418(1998) 345, Erratum-ibid. Phys.Lett.429 (1998) 414.

[10] A.D. Martin and M.G. Ryskin, Phys.Rev. D57 (1998) 6692.

[11] L.Mankiewicz, G. Piller and T. Weigl, Eur. Phys. J. C5 (1998) 119.

[12] P.A.M. Guichon and M. Vanderhaeghen, Prog. Part. Nucl. Phys. 21 (1998) 125, M. Vanderhaeghen, P.A.M. Guichon, M. Guidal Phys. Rev. Lett. 80 (1998) 5064.

[13] A.V. Radyushkin,Few Body Syst.Suppl.99 (1998)1.

[14] P. Kroll, Proceeding of ICTP Conference on Perspectives in Hadronic Physics, Trieste, Italy, 12-16 May 1997 in press; e-Print Archive: hep-ph@xxx.lanl.gov - 9709426

[15] M. Eides, L. Frankfurt and M. Strikman, hep-ph/9809277

[16] L. Frankfurt, W. Koepf and M. Strikman, Phys. Rev. D54 (1996) 3194.

[17] S.J.Brodsky, L.L.Frankfurt, J.F.Gunion, A.H.Mueller, and M.Strikman, Phys.Rev.D50 (1994) 3134 
[18] L.L. Frankfurt, M.V. Polyakov, M. Strikman, hep-ph/9808449.

[19] M. Penttinen, M.V. Polyakov and K. Goeke, Bochum University preprint, RUBTPII-19/98

[20] V. Y. Petrov et al., Phys. Rev. D57 (1998) 4325

[21] D. Diakonov, V. Petrov, P. Pobylitsa, M. Polyakov, and C. Weiss, Nucl. Phys. B480 (1996) 341

[22] D.I.Diakonov, Yu.V.Petrov and P.V.Pobylitsa, Nucl. Phys. B306 (1988) 809

[23] V.Yu. Petrov and P.V. Pobylitsa, hep-ph/9712203.

[24] V.Yu. Petrov et al., Bochum University preprint RUB-TPII-8/98, hep-ph/9807229, Phys. Rev. D, in print

[25] X. Ji, W. Melnitchouk and X.Song, Phys. Rev. D56 (1997) 1

[26] L. Mankiewicz, G. Piller and A. Radyushkin, preprint hep-ph/9812467. 


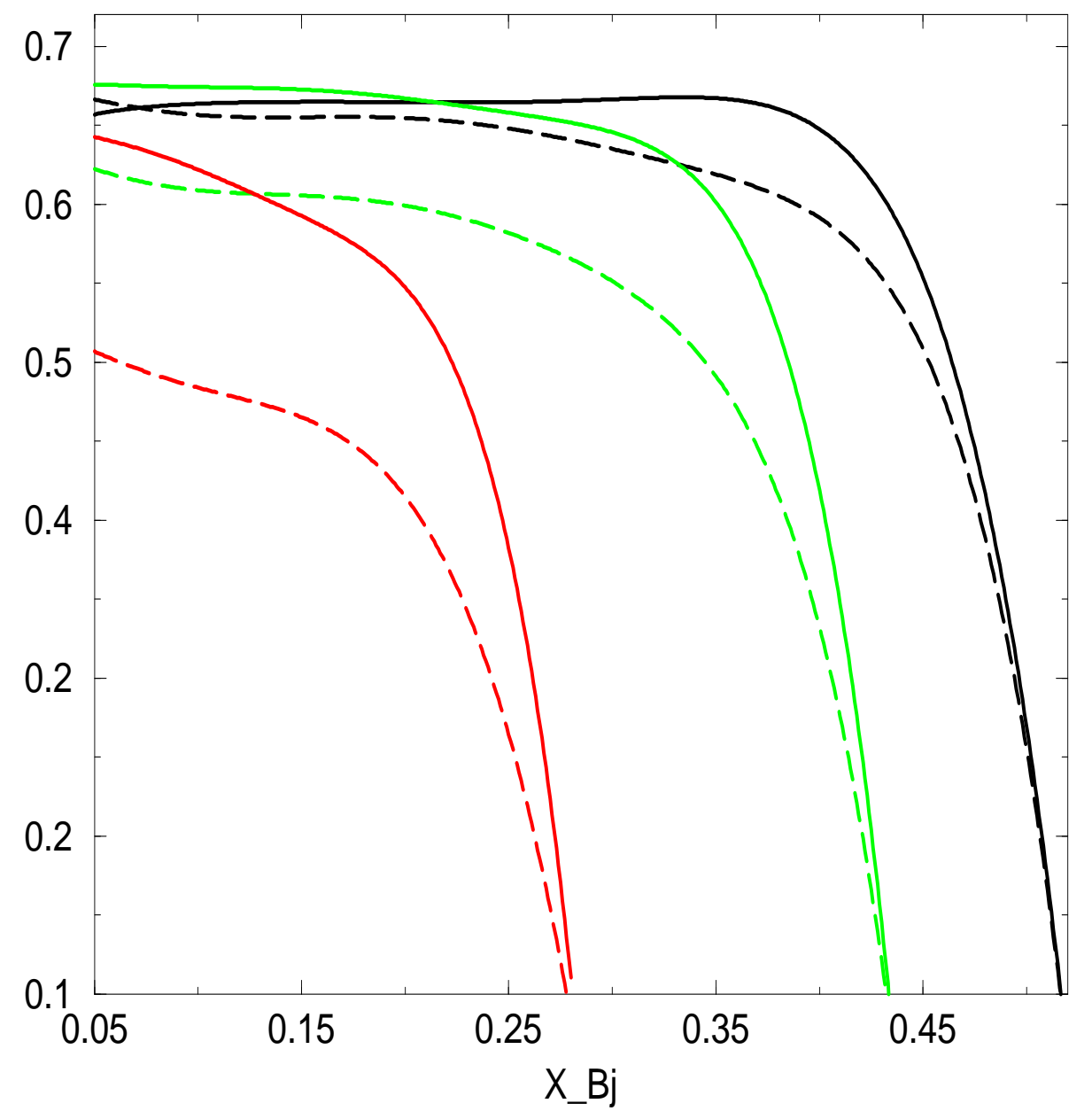

Figure 1: The asymmetry $\mathcal{A}_{+}$as a function of Bjorken $x$ at $t=-0.1,-0.3,-0.5 \mathrm{GeV}^{2}$. Solid lines: With asymptotic pion distribution amplitude $\eta=1$ Dashed lines: With Chernyak-Zhitnitsky pion distribution amplitude $\eta=5 / 3$ 